\title{
Inhibition of Trypanosoma cruzi Hexokinase
}

\section{by Bisphosphonates}

Michael P. Hudock\#, C. E. Sanz-Rodríquez†, Yongcheng Song\$, Julian M. W. Chan§, Yonghui Zhang§, Sarah Odeh\$, Thomas Kosztowski§, Annette Leon-Rossell\#, J. L.

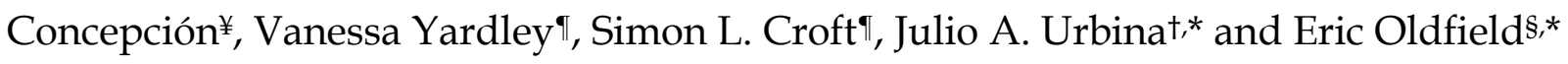

\section{Supporting Information}




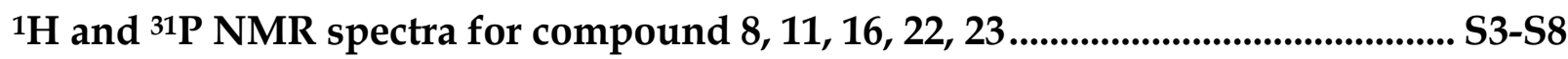

Table S1: Analytical Data for Bisphosphonates

\begin{tabular}{|c|c|c|c|c|c|c|c|}
\hline \multirow[t]{2}{*}{ Cpd } & \multirow{2}{*}{$\begin{array}{l}\text { Molecular } \\
\text { Formula }\end{array}$} & \multicolumn{3}{|c|}{ Calculated } & \multicolumn{3}{|c|}{ Found } \\
\hline & & $\begin{array}{c}\text { Carbon } \\
(\%)\end{array}$ & $\begin{array}{c}\text { Hydrogen } \\
(\%)\end{array}$ & $\begin{array}{c}\text { Nitrogen } \\
(\%)\end{array}$ & $\begin{array}{c}\text { Carbon } \\
(\%)\end{array}$ & $\begin{array}{c}\text { Hydrogen } \\
(\%)\end{array}$ & $\begin{array}{c}\text { Nitrogen } \\
(\%)\end{array}$ \\
\hline 8 & $\mathrm{C}_{11} \mathrm{H}_{18} \mathrm{~N}_{2} \mathrm{O}_{9} \mathrm{P}_{2}$ & 34.39 & 4.72 & 7.29 & 34.35 & 4.20 & 7.18 \\
\hline 11 & $\begin{array}{l}\mathrm{C}_{15} \mathrm{H}_{15} \mathrm{~F}_{2} \mathrm{O}_{7} \mathrm{P}_{2} \mathrm{Na} \\
-\mathrm{H}_{2} \mathrm{O} \\
\end{array}$ & 40.20 & 3.82 & 0.00 & 39.91 & 3.91 & 0.00 \\
\hline 16 & $\begin{array}{l}\mathrm{C}_{13} \mathrm{H}_{14} \mathrm{FNO}_{6} \mathrm{P}_{2} \bullet \\
\mathrm{H}_{2} \mathrm{O}\end{array}$ & 41.18 & 4.25 & 3.69 & 41.03 & 4.15 & 3.66 \\
\hline 22 & $\mathrm{C}_{9} \mathrm{H}_{16} \mathrm{~N}_{2} \mathrm{O}_{7} \mathrm{P}_{2}$ & 33.14 & 4.94 & 8.59 & 32.68 & 4.51 & 8.12 \\
\hline 23 & $\mathrm{C}_{13} \mathrm{H}_{14.8} \mathrm{NPO}_{3.4}$ & 57.74 & 5.52 & 5.18 & 57.81 & 5.85 & 5.19 \\
\hline 24 & $\mathrm{C}_{10} \mathrm{H}_{17} \mathrm{NO}_{7} \mathrm{P}_{2}$ & 36.94 & 5.27 & 4.31 & 36.54 & 5.36 & 4.25 \\
\hline 25 & $\mathrm{C}_{17} \mathrm{H}_{17} \mathrm{O}_{5} \mathrm{PNa}_{2}$ & 46.27 & 5.48 & 0.00 & 46.47 & 5.18 & 0.00 \\
\hline 27 & $\begin{array}{l}\mathrm{C}_{13} \mathrm{H}_{15} \mathrm{NO}_{8} \mathrm{P}_{2} \bullet \\
0.5 \mathrm{H}_{2} \mathrm{O}\end{array}$ & 40.64 & 4.20 & 3.65 & 41.11 & 4.02 & 3.77 \\
\hline 28 & $\begin{array}{l}\mathrm{C}_{12} \mathrm{H}_{14} \mathrm{~N}_{2} \mathrm{O}_{7} \mathrm{P}_{2} \bullet \\
1.5 \mathrm{H}_{2} 0\end{array}$ & 37.22 & 4.43 & 7.23 & 37.32 & 4.15 & 7.11 \\
\hline 39 & $\mathrm{C}_{6} \mathrm{H}_{10} \mathrm{~N}_{2} \mathrm{O}_{7} \mathrm{P}_{2}$ & 25.37 & 3.55 & 9.86 & 25.21 & 3.40 & 9.61 \\
\hline 42 & $\mathrm{C}_{7} \mathrm{H}_{11} \mathrm{~N}_{2} \mathrm{P}_{2} \mathrm{O}_{7} \mathrm{Na}$ & 27.62 & 3.65 & 9.21 & 27.90 & 4.19 & 9.08 \\
\hline
\end{tabular}




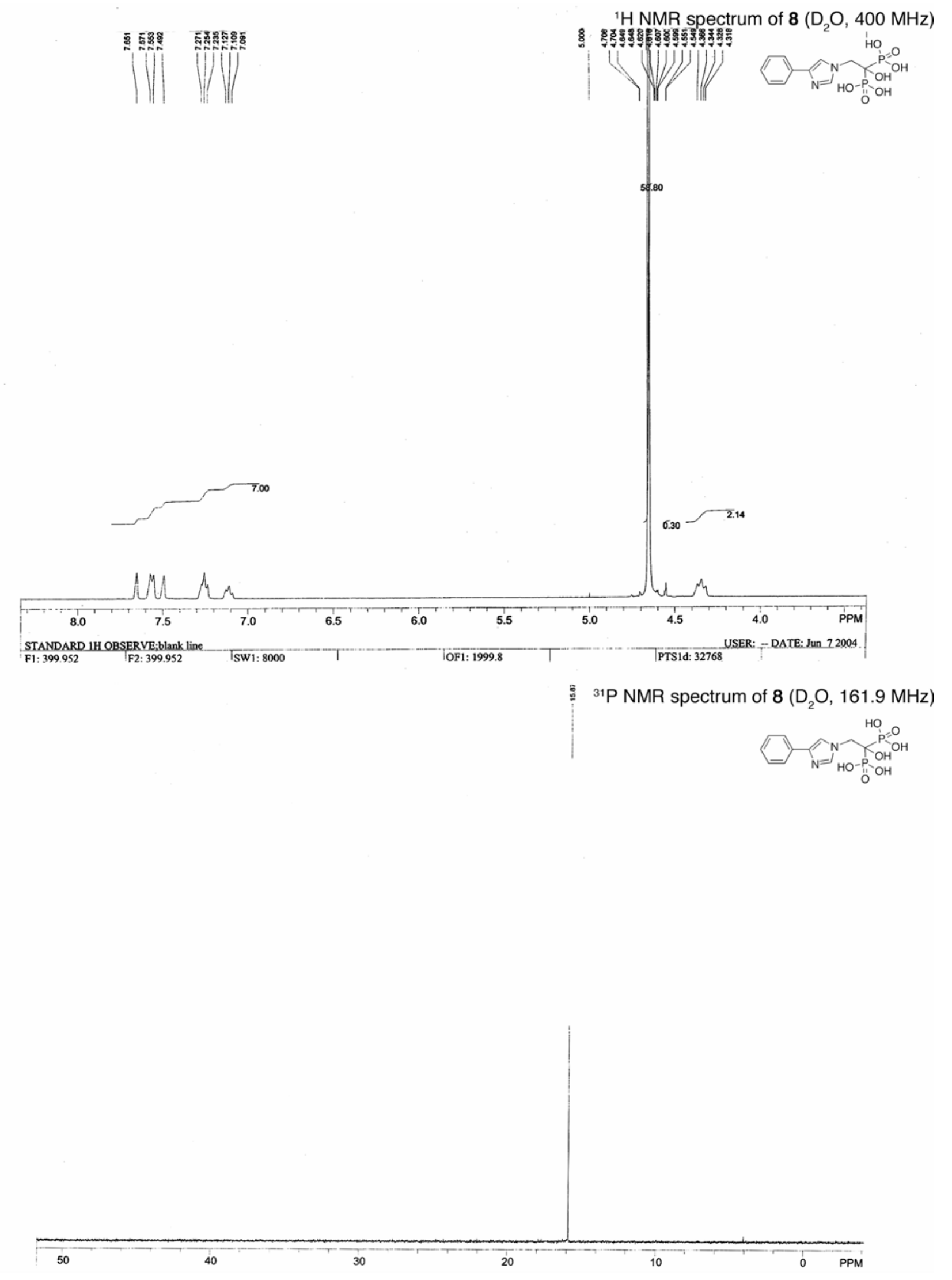


${ }^{1} \mathrm{H}$ NMR spectrum of $11\left(\mathrm{D}_{2} \mathrm{O}, 400 \mathrm{MHz}\right)$

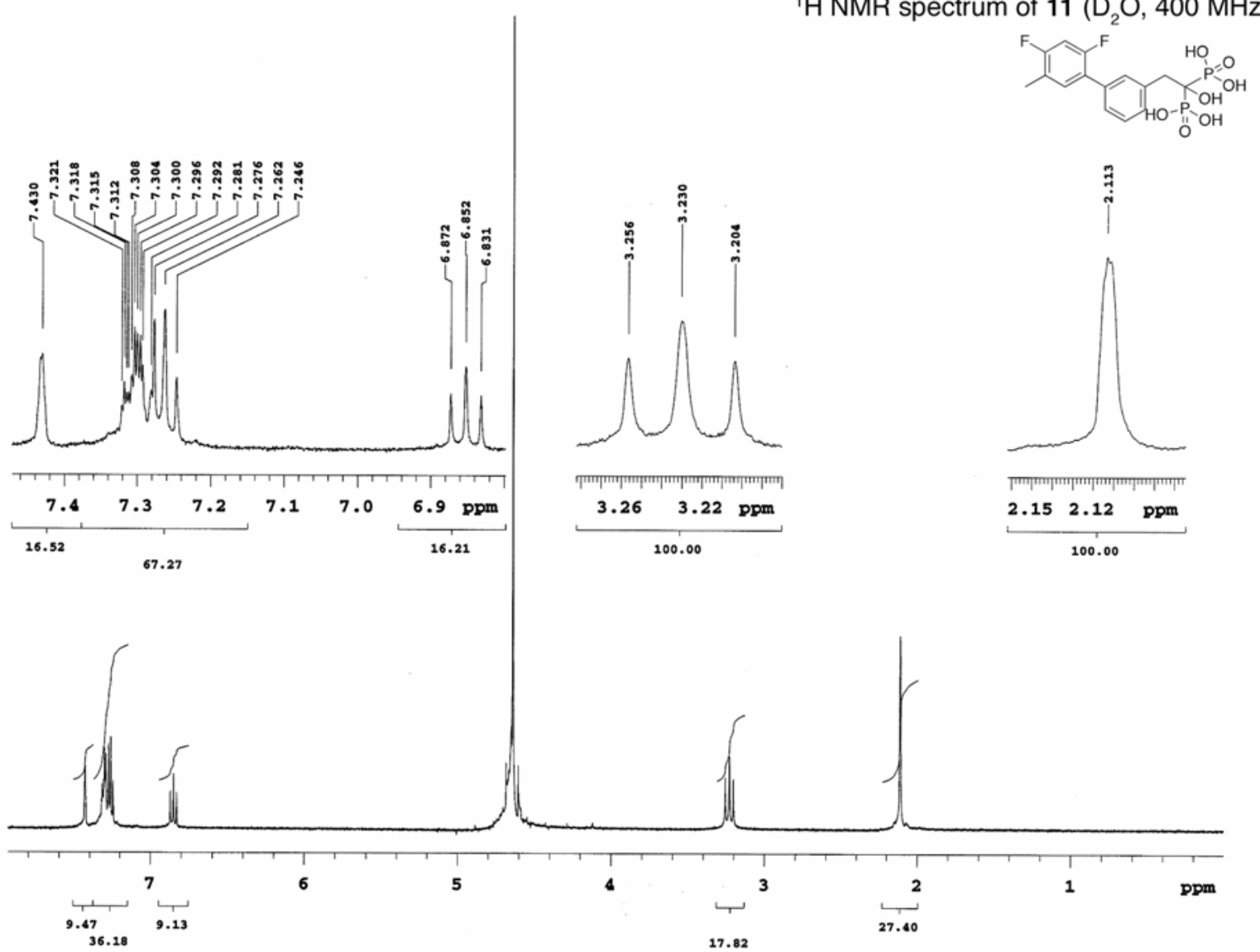

${ }^{31} \mathrm{P}$ NMR spectrum of $11\left(\mathrm{D}_{2} \mathrm{O}, 161.9 \mathrm{MHz}\right)$
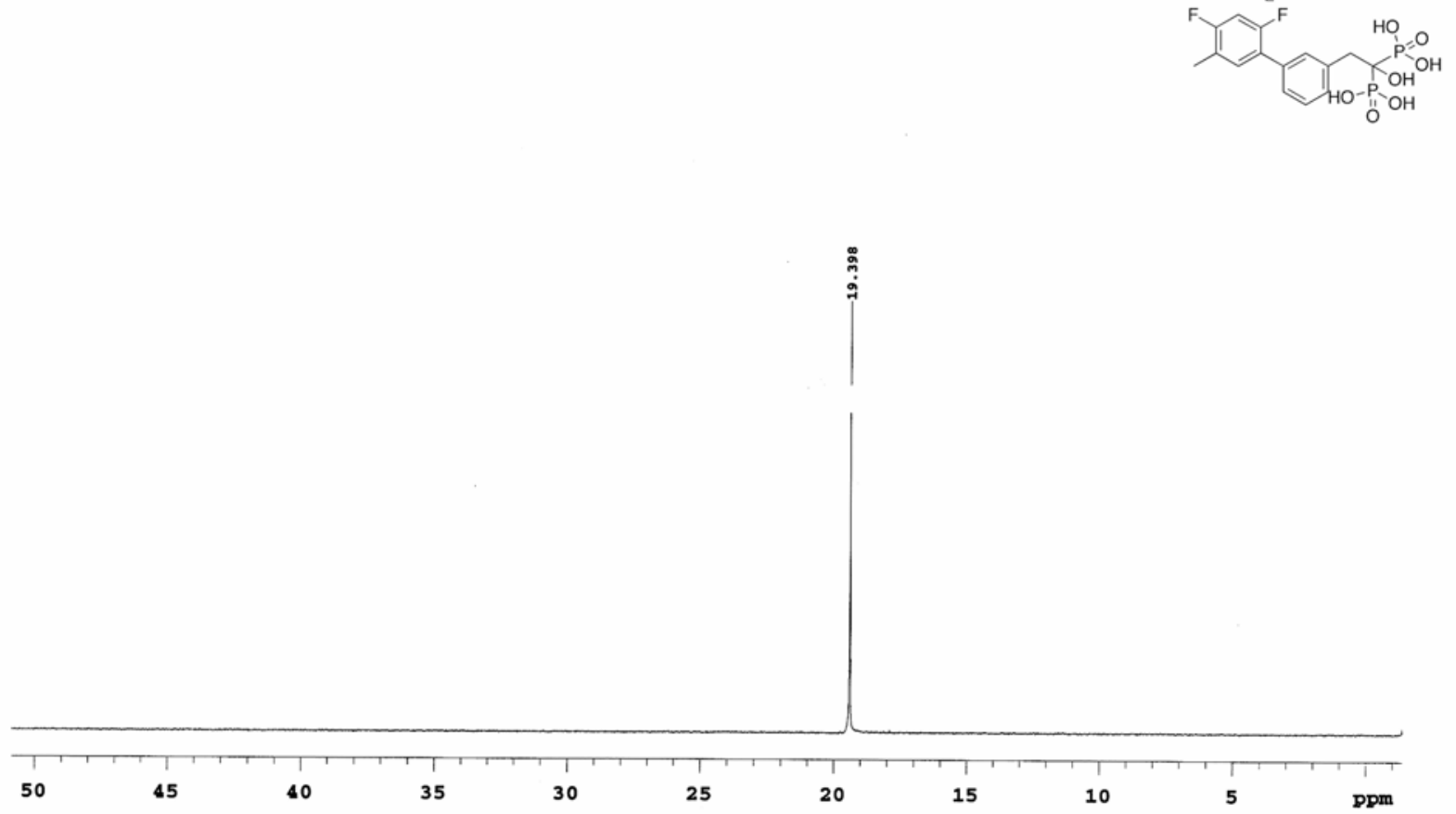
${ }^{19} \mathrm{~F}$ NMR Spectrum for $11\left(\mathrm{D}_{2} \mathrm{O}, 376.9 \mathrm{MHz}\right)$
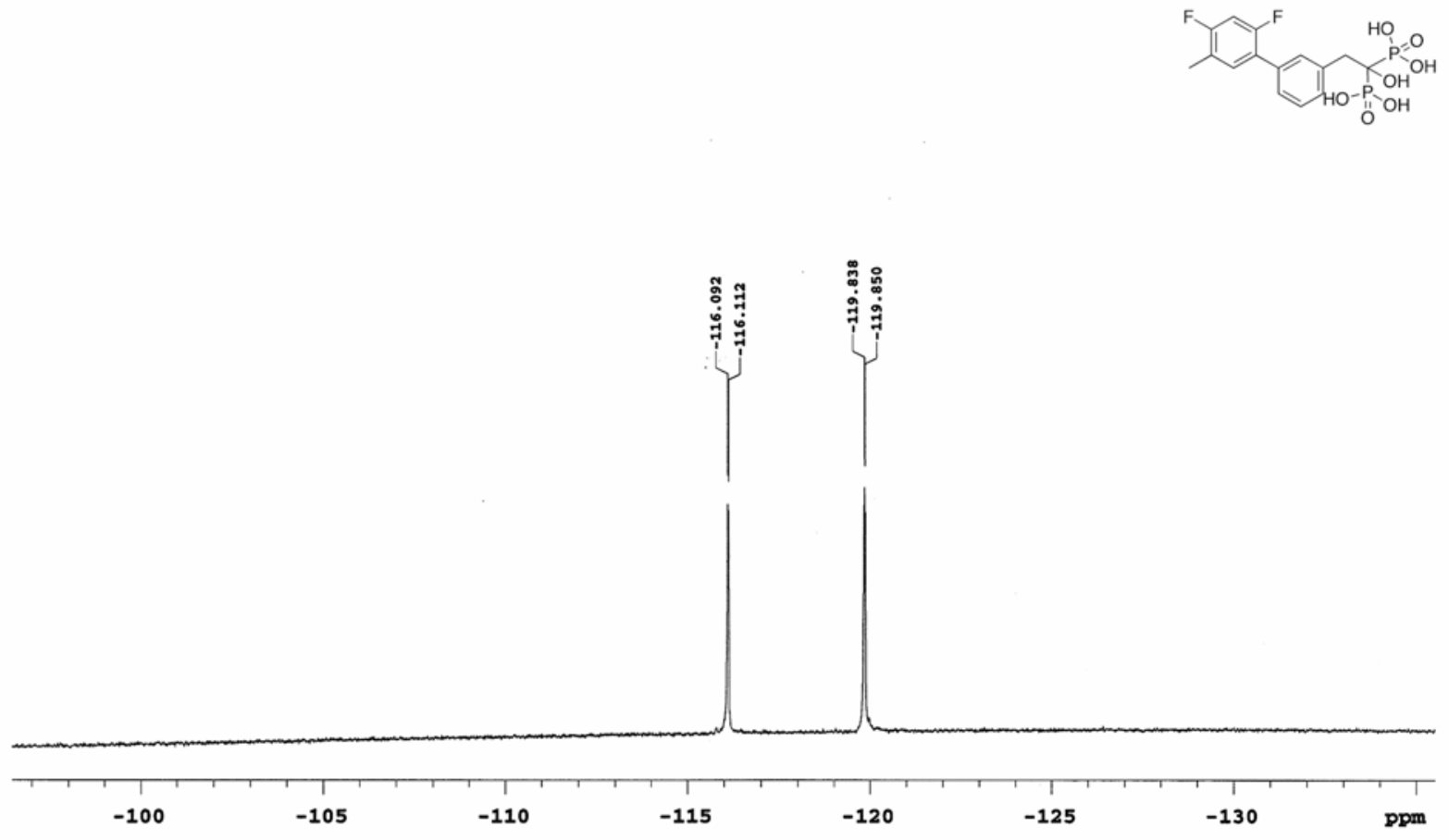

${ }^{1} \mathrm{H}$ NMR Spectrum of $16\left(\mathrm{D}_{2} \mathrm{O}, 400 \mathrm{MHz}\right)$
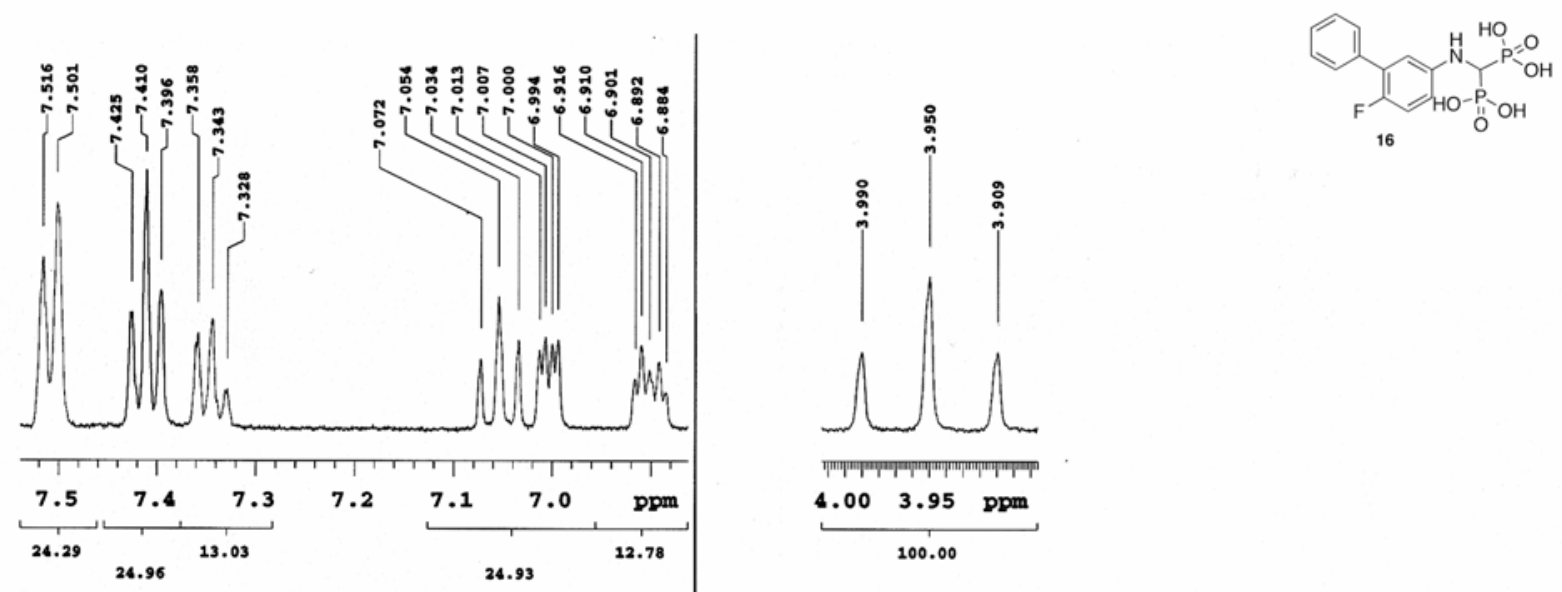

100.00

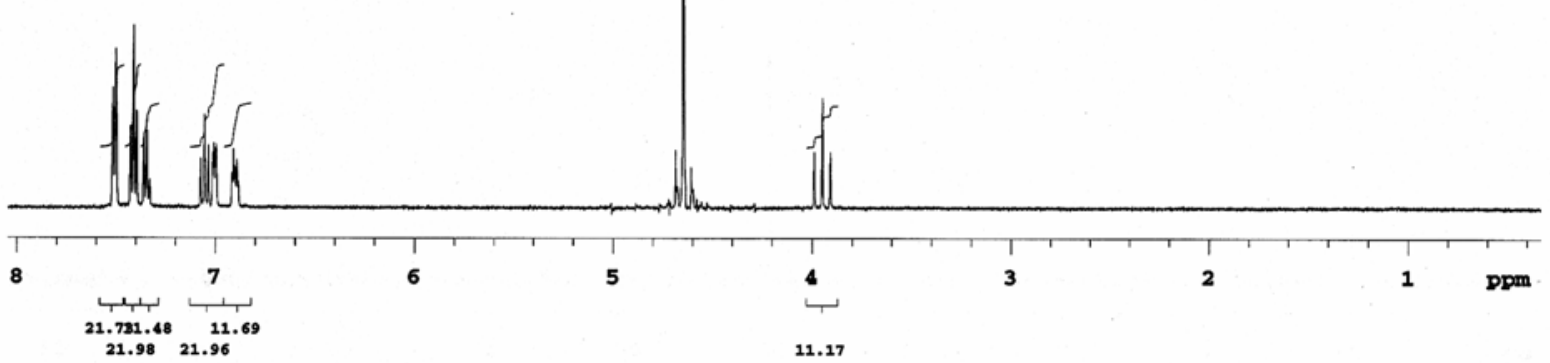


${ }^{31} \mathrm{P}$ NMR Spectrum of $16\left(\mathrm{D}_{2} \mathrm{O}, 161.9 \mathrm{MHz}\right)$
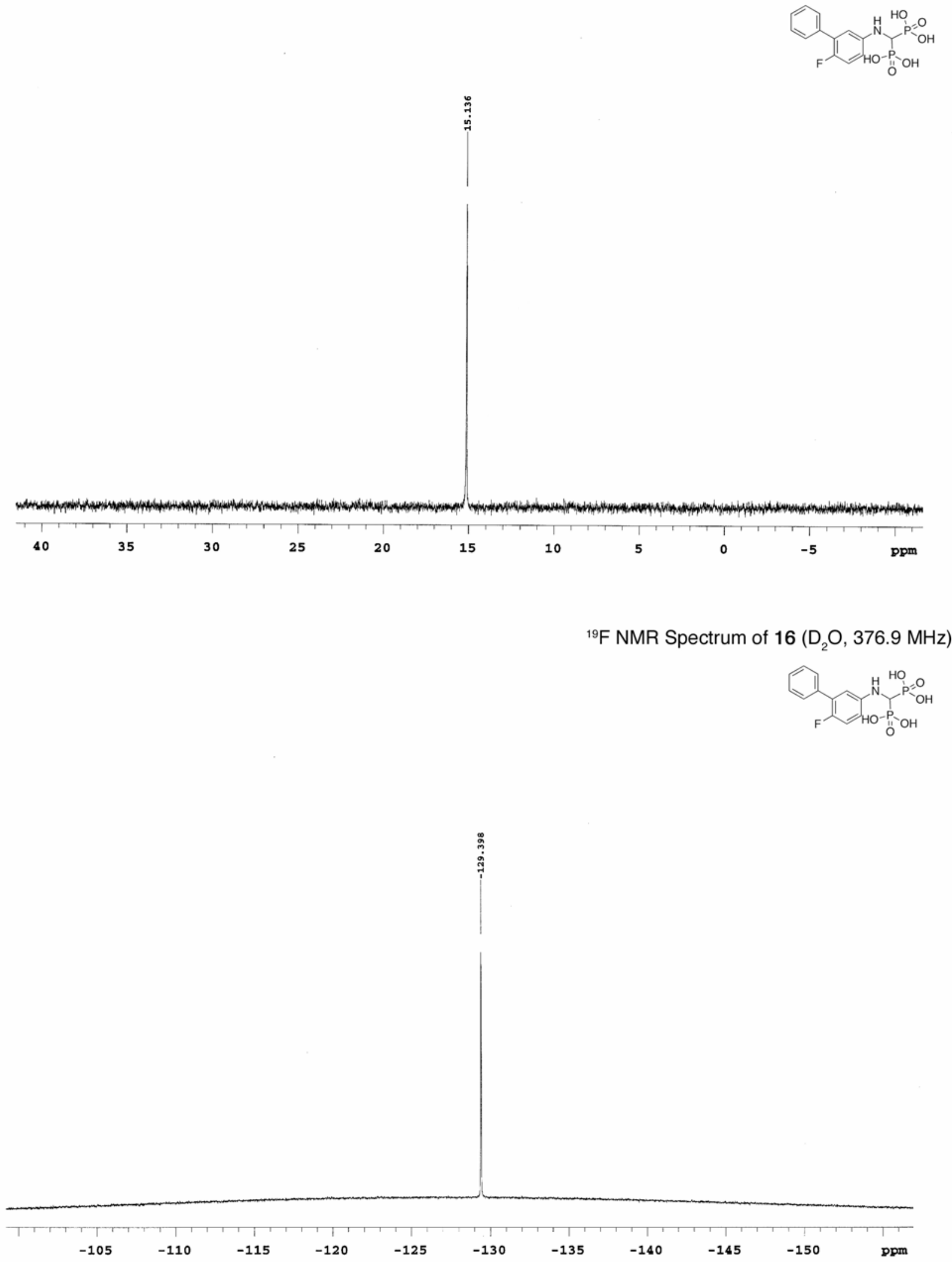


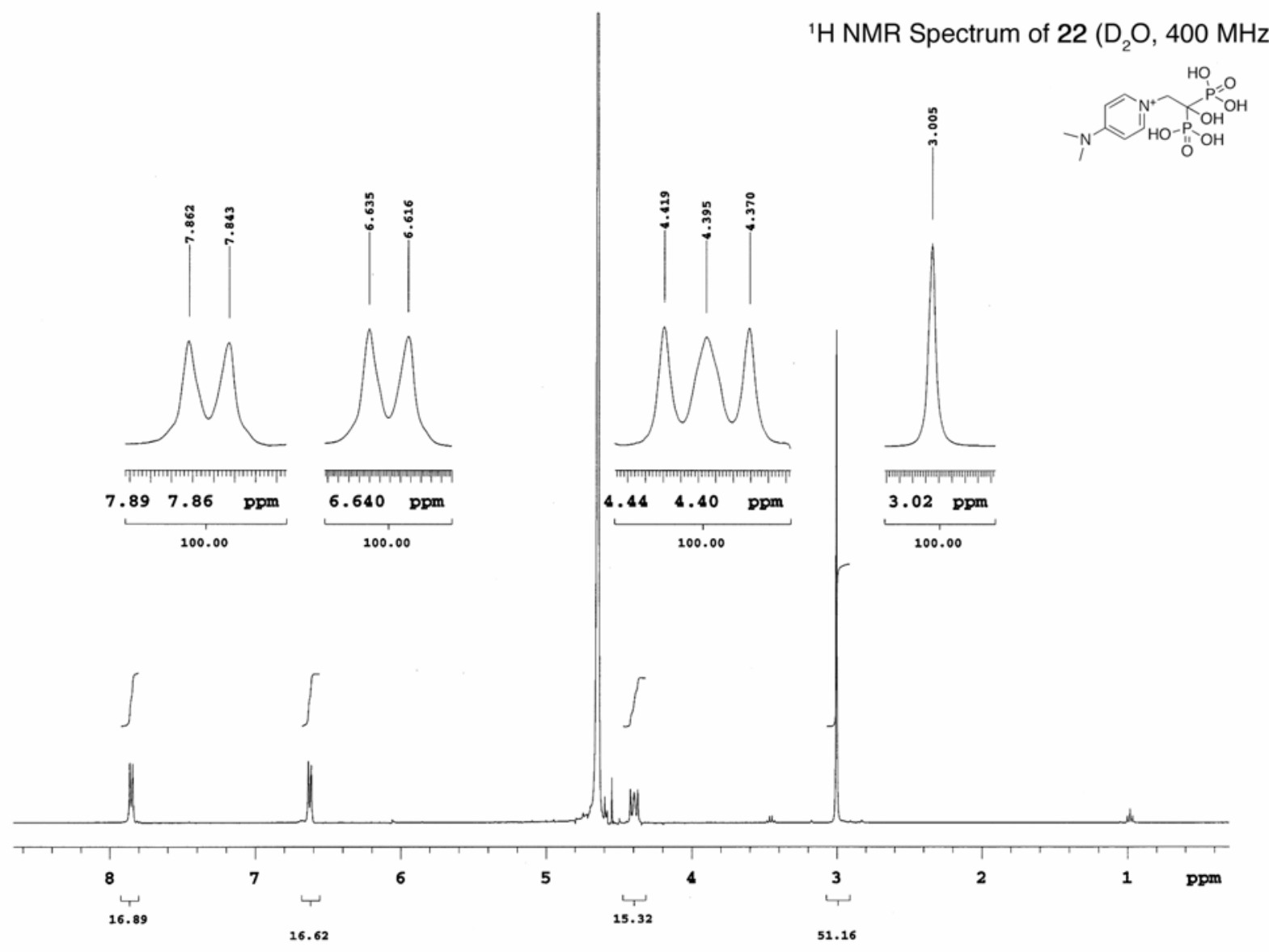

${ }^{31} \mathrm{P}$ NMR Spectrum of $22\left(\mathrm{D}_{2} \mathrm{O}, 161.9 \mathrm{MHz}\right)$
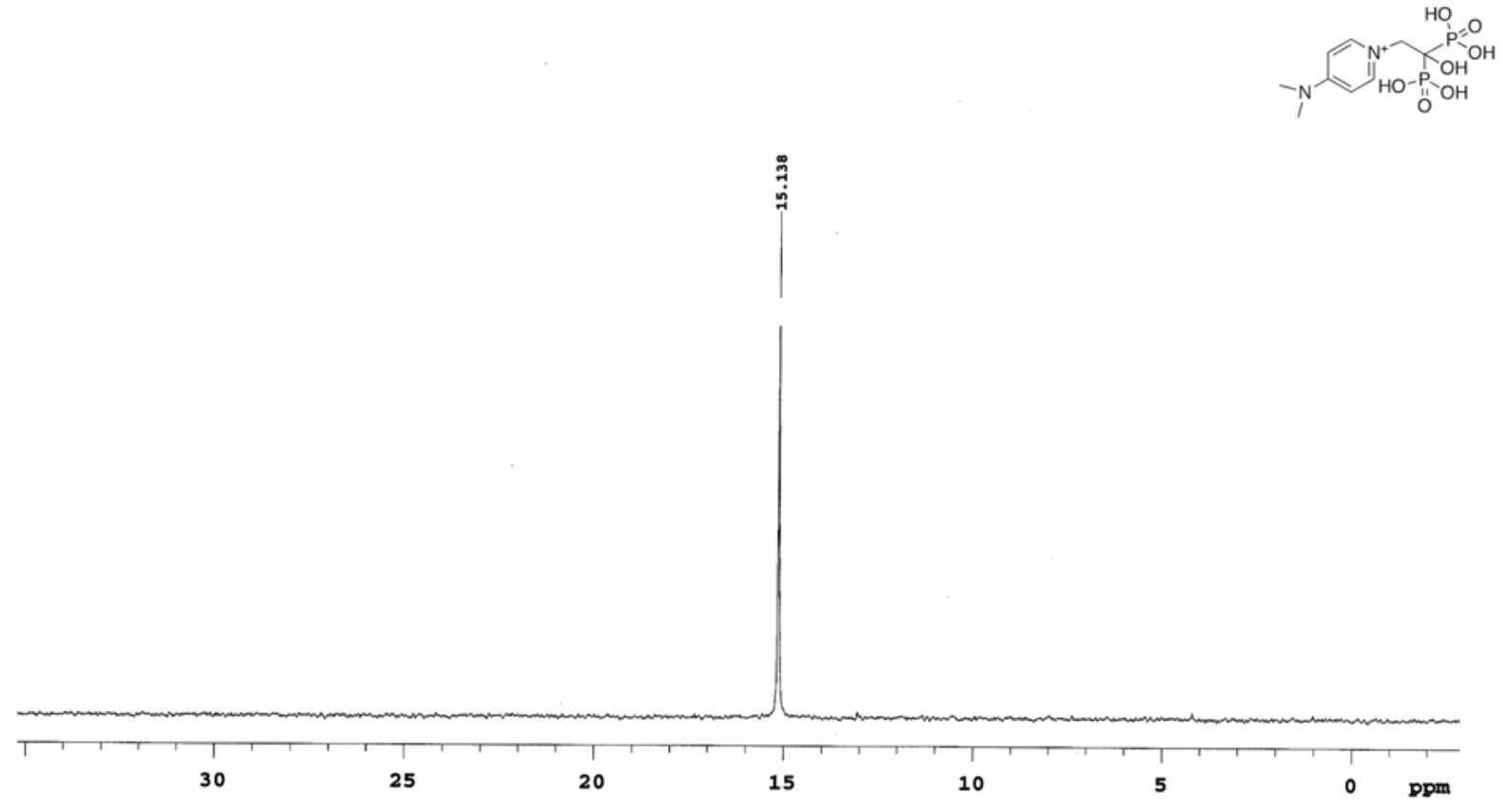


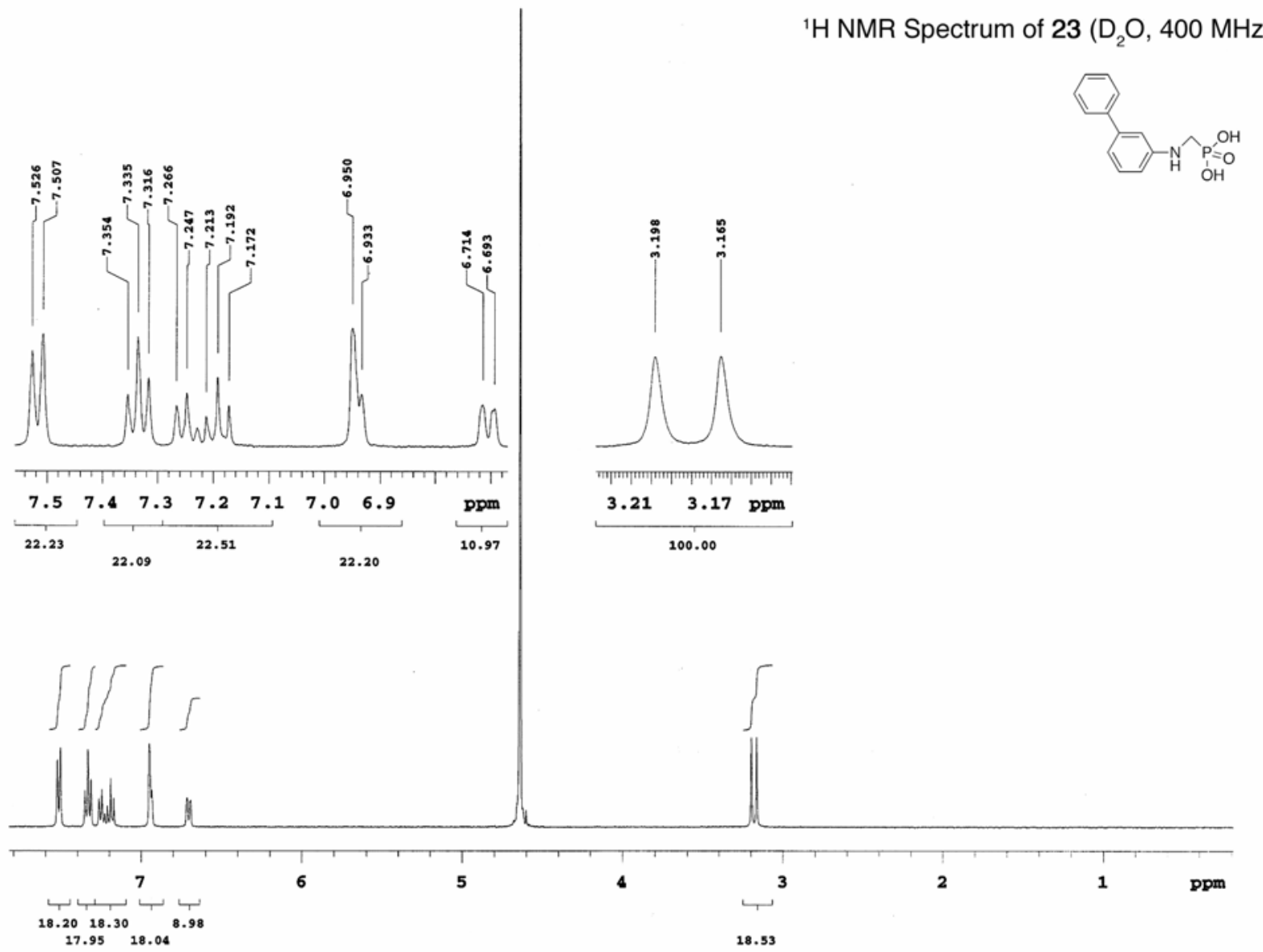

${ }^{31} \mathrm{P}$ NMR Spectrum of $23\left(\mathrm{D}_{2} \mathrm{O}, 161.9 \mathrm{MHz}\right)$

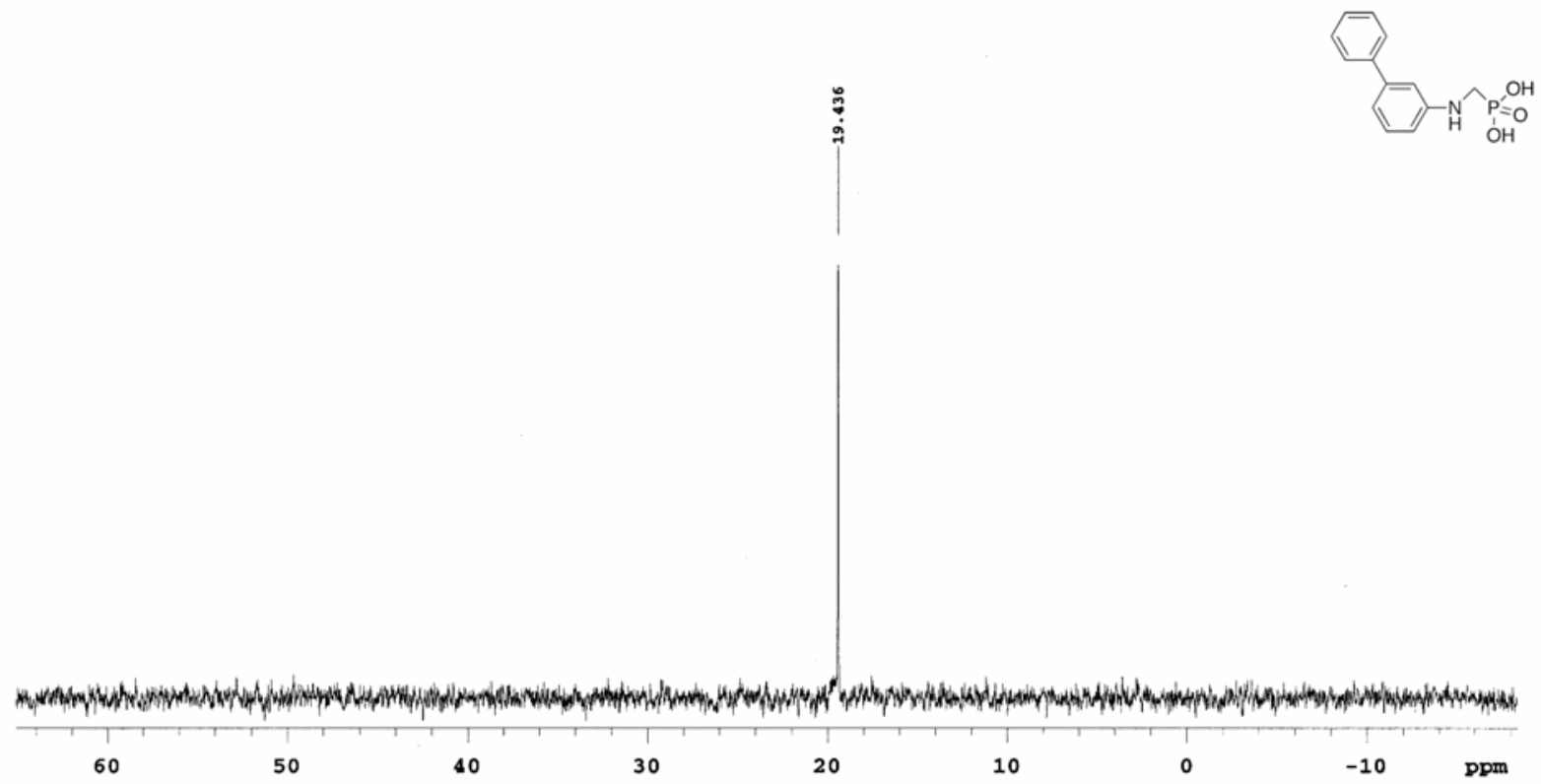

